\title{
The dopaminergic reward system underpins gender differences in social preferences
}

\author{
Alexander Soutschek ${ }^{1 \star}$, Christopher J. Burke ${ }^{1}$, Anjali Raja Beharelle ${ }^{1}$, Robert Schreiber1, \\ Susanna C. Weber1, Iliana I. Karipidis ${ }^{1,2,3}$, Jolien ten Velden ${ }^{1,4}$, Bernd Weber (1) 5,6 , Helene Haker ${ }^{7}$, \\ Tobias Kalenscher ${ }^{8}$ and Philippe N. Tobler ${ }^{1,3}$
}

\begin{abstract}
Women are known to have stronger prosocial preferences than men, but it remains an open question as to how these behavioural differences arise from differences in brain functioning. Here, we provide a neurobiological account for the hypothesized gender difference. In a pharmacological study and an independent neuroimaging study, we tested the hypothesis that the neural reward system encodes the value of sharing money with others more strongly in women than in men. In the pharmacological study, we reduced receptor type-specific actions of dopamine, a neurotransmitter related to reward processing, which resulted in more selfish decisions in women and more prosocial decisions in men. Converging findings from an independent neuroimaging study revealed gender-related activity in neural reward circuits during prosocial decisions. Thus, the neural reward system appears to be more sensitive to prosocial rewards in women than in men, providing a neurobiological account for why women often behave more prosocially than men.
\end{abstract}

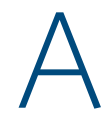
large body of evidence suggests that women are often more prosocial (for example, generous, altruistic and inequality averse) than men, at least when other factors such as reputation and strategic considerations are excluded ${ }^{1-3}$. This dissociation could result from cultural expectations and gender stereotypes, because in Western societies women are more strongly expected to be prosocial ${ }^{4-6}$ and sensitive to variations in social context than $\operatorname{men}^{1}$. It remains an open question, however, whether and how on a neurobiological level the social preferences of women and men arise from differences in brain functioning. The assumption of gender differences in social preferences predicts that the neural reward system's sensitivity to prosocial and selfish rewards should differ between women and men. Specifically, the hypothesis would be that the neural reward system is more sensitive to prosocial than selfish rewards in women and more sensitive to selfish than prosocial rewards in men. The goal of the current study was to test in two independent experiments for the hypothesized gender differences on both a pharmacological and a haemodynamic level. In particular, we examined the functions of the neurotransmitter dopamine using a dopamine receptor antagonist, and the role of the striatum (a brain region strongly innervated by dopamine neurons) during social decision-making in women and men using neuroimaging.

The neurotransmitter dopamine is thought to play a key role in neural reward processing ${ }^{7,8}$. Recent evidence suggests that dopaminergic activity is sensitive not only to rewards for oneself but to rewards for others as well ${ }^{9}$. The assumption that dopamine is sensitive to both self- and other-related outcomes is consistent with the finding that the striatum shows activation for both selfish and shared rewards ${ }^{10-15}$. The dopaminergic response may represent a net signal encoding the difference between the value of preferred and unpreferred rewards ${ }^{8}$. Regarding the hypothesized gender differences in social preferences, this account makes the following predictions. If women prefer shared (prosocial) outcomes ${ }^{2}$, women's dopaminergic signals to shared rewards will be stronger than to non-shared (selfish) rewards, so reducing dopaminergic activity should bias women to make more selfish decisions. In line with this hypothesis, a functional imaging study reported enhanced striatal activation in female participants during charitable donations ${ }^{11}$. In contrast, if men prefer selfish over prosocial rewards, dopaminergic activity should be enhanced to selfish compared to prosocial rewards. In line with this view, upregulating dopaminergic activity in a sample of exclusively male participants increased selfish behaviour in a bargaining game $^{16}$. Thus, contrary to the hypothesized effect in women, reducing dopaminergic neurotransmission should render men more prosocial. Taken together, the current study tested the following three predictions: we expected the dopaminergic reward system (1) to be more sensitive to prosocial than selfish rewards in women and (2) to be more sensitive to selfish than prosocial rewards in men. As a consequence of these two predictions, we also predicted (3) dopaminoceptive regions such as the striatum to show stronger activation to prosocial relative to selfish rewards in women than in men.

To test these predictions, we conducted a pharmacological study in which we reduced dopaminergic neurotransmission with amisulpride. Amisulpride is a dopamine antagonist that is highly specific for dopaminergic D2/D3 receptors ${ }^{17}$. After receiving amisulpride or placebo, participants performed an interpersonal decision task ${ }^{18-20}$, in which they made choices between a monetary reward only for themselves (selfish reward option) and sharing money with others

\footnotetext{
'Laboratory for Social and Neural Systems Research, Department of Economics, University of Zurich, 8006 Zurich, Switzerland. ${ }^{2}$ Department of Child and Adolescent Psychiatry and Psychotherapy, Psychiatric Hospital, University of Zurich, 8032 Zurich, Switzerland. ${ }^{3}$ Neuroscience Center Zurich, University of Zurich, Swiss Federal Institute of Technology Zurich, 8057 Zurich, Switzerland. ${ }^{4}$ Max-Planck-Institute for Psycholinguistics,

6525 XD Nijmegen, Netherlands. ${ }^{5}$ Department of Epileptology, University Hospital Bonn, 53105 Bonn, Germany. ${ }^{6}$ Center for Economics and Neuroscience, University of Bonn, 53127 Bonn, Germany. ${ }^{7}$ Translational Neuromodeling Unit, Institute for Biomedical Engineering, University of Zurich and ETH Zurich, 8032 Zurich, Switzerland. ${ }^{8}$ Comparative Psychology, Institute of Experimental Psychology, Heinrich Heine University, 40225 Düsseldorf, Germany.

*e-mail: alexander.soutschek@econ.uzh.ch
} 
(prosocial reward option). We expected that blocking dopaminergic neurotransmission with amisulpride, relative to placebo, would result in fewer prosocial choices in women and more prosocial choices in men. To investigate whether potential gender-related effects of dopamine are selective for social decision-making, we also tested the effects of amisulpride on time preferences in a non-social control task that was matched to the interpersonal decision task in terms of choice structure.

In addition, because dopaminergic neurotransmission plays a crucial role in brain regions involved in value processing, such as the striatum ${ }^{21}$, a gender-related role of dopaminergic activity for social decision-making should also be reflected by dissociable activity patterns in the striatum. Therefore, to further test our hypothesis, we investigated the neural correlates of social decision-making in a functional imaging study. In line with our predictions for the pharmacological study, we expected to find stronger striatum activity during prosocial relative to selfish decisions in women, whereas men should show enhanced activity in the striatum for selfish relative to prosocial choices.

\section{Results}

We conducted a randomized, double-blind, placebo-controlled, crossover study. In two consecutive sessions, female and male participants either received the selective D2/D3 antagonist amisulpride in session 1 and placebo in session 2 , or vice versa (Fig. 1a). In both sessions, participants performed an interpersonal decision task and a non-social intertemporal control task (in counterbalanced order).

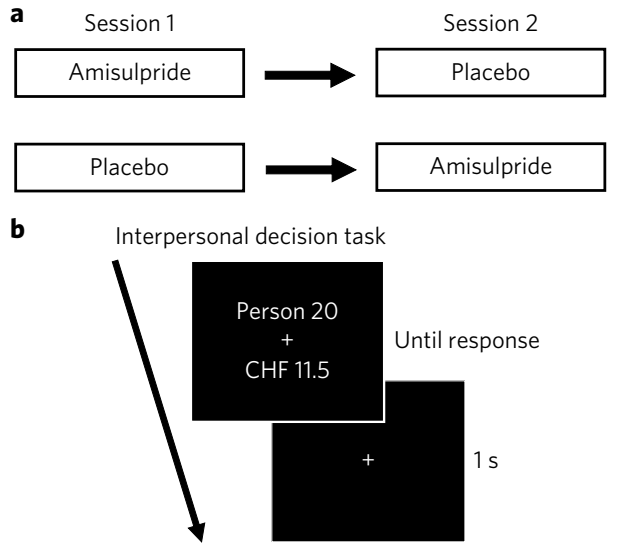

c

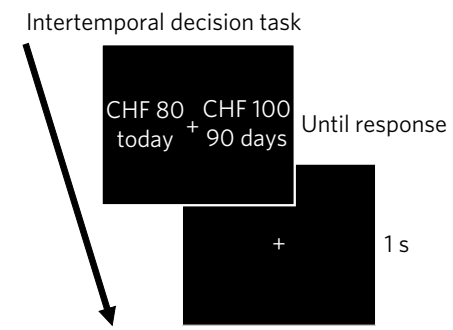

Fig. 1 | Study design and experimental tasks. a, Participants performed both the interpersonal and intertemporal decision tasks in two sessions. In session 1, participants received either the dopamine antagonist amisulpride or placebo, and in session 2 (14 days after session 1) the respective other substance. $\mathbf{b}$, In the interpersonal decision task, participants made choices between a prosocial reward option (CHF7.5 for the participant and for the person at the indicated social distance) and a selfish reward option (only the participant receives money, for example, CHF11.5). c, In the intertemporal decision task, participants chose between a smaller-sooner (for example, CHF100 today) and a larger-later (for example, CHF300 in 90 days) reward option.
In the interpersonal decision task (Fig. 1b), participants made choices between a selfish reward only for themselves (7.5-15.5 Swiss francs $(\mathrm{CHF})$ ) and a prosocial reward that was equally shared between themselves and a person at varying social distances (CHF7.5 both for the participant and for other). The social distance of the other person ranged from very close to being a stranger. In the intertemporal decision task (Fig. 1c), participants chose between smallersooner (SS) rewards (for example, CHF80 today) and larger-later (LL) rewards (for example, CHF100 in 90 days). Both the interpersonal and intertemporal decision task measure how the subjective value of a reward option is discounted as a function of (social or temporal) distance. These tasks thus allowed a comparison of the gender-specific effects of the pharmacological intervention on social relative to individual decision-making, while keeping the choice structure of the used tasks constant.

Female and male participants were matched for baseline measures that might potentially influence drug effects on the dopaminergic system, including age ( $t<1, P=0.32$, Cohen's $d=0.24)$, body mass index (BMI) $(t<1.42, P=0.16$, Cohen's $d=0.39)$ and working memory capacity (digit span forward: $t<1, P=0.93$, Cohen's $d=0.03$; digit span backward: $t<1.21, P=0.23$, Cohen's $d=0.33$ ). In addition, there was no evidence that amisulpride changed participants' self-reported mood, alertness or calmness during the experiment (all $F<1.75$, all $P>0.19$, all partial eta squared, $\eta_{\mathrm{p}}{ }^{2}<0.040$ ). This suggests that potential drug effects on task performance cannot be attributed to drug-related mood changes.

Reducing dopaminergic activity differentially modulates social preferences in men and women. In the interpersonal decision task under placebo, female subjects chose the prosocial reward option more often than male subjects $(t(53)=2.01, P=0.049$, Cohen's $d=0.55$ ), which is consistent with the findings that women have stronger preferences for shared outcomes than $\operatorname{men}^{2,22}$. To test the role of dopaminergic neurotransmission in prosocial and selfish decision-making, we examined the effects of amisulpride on choices between sharing and non-sharing in female and male participants. We computed a mixed-measures analysis of variance (ANOVA) on prosocial decisions (\% sharing of all decisions) including the within-participant factor Substance (amisulpride versus placebo) and the between-participant factors Gender (female versus male) and Substance Order (amisulpride-placebo versus placeboamisulpride). Only the Substance $\times$ Gender interaction yielded a significant effect $\left(F(1,51)=10.92, P=0.002, \eta_{\mathrm{p}}{ }^{2}=0.176\right)$, indicating dissociable effects of dopamine on social decision-making in female and male participants (all other effects $F<2.2$, all $P>0.14$, all $\left.\eta_{\mathrm{p}}{ }^{2}<0.019\right)$. Planned comparisons revealed that females made fewer prosocial choices under amisulpride $(45 \%)$ than under placebo (51\%) $(t(26)=2.64, P=0.014$, Cohen's $d=0.52)$. In contrast, males made more prosocial choices in the amisulpride (44\%) than in the placebo condition $(40 \%)(t(27)=2.29, P=0.030$, Cohen's $d=0.43)$. Note that this result pattern is at variance with the possibility that choices have become more random under amisulpride because females' choices were closer to the $50 \%$ level under placebo than under amisulpride. Importantly, the data confirm the hypothesized gender-related role of dopamine in social decision-making (Fig. 2a and Supplementary Fig. 1a,b).

Because females were more prosocial than males in the placebo condition, one might wonder whether the drug effects on social decision-making depend on individual differences in baseline prosociality rather than on gender. To control for this potential confounder, we entered the number of prosocial decisions under placebo as a covariate in the above reported ANOVA. This analysis also yielded a significant Substance $\times$ Gender interaction $\left(F(1,50)=8.55, P=0.005, \eta_{\mathrm{p}}^{2}=0.146\right)$, but provided no evidence for an impact of baseline prosociality on drug effects $(F(1,50)=1.72$, $P=0.20, \eta_{\mathrm{p}}{ }^{2}=0.033$ ) (Supplementary Fig. 1c,d). Moreover, the 

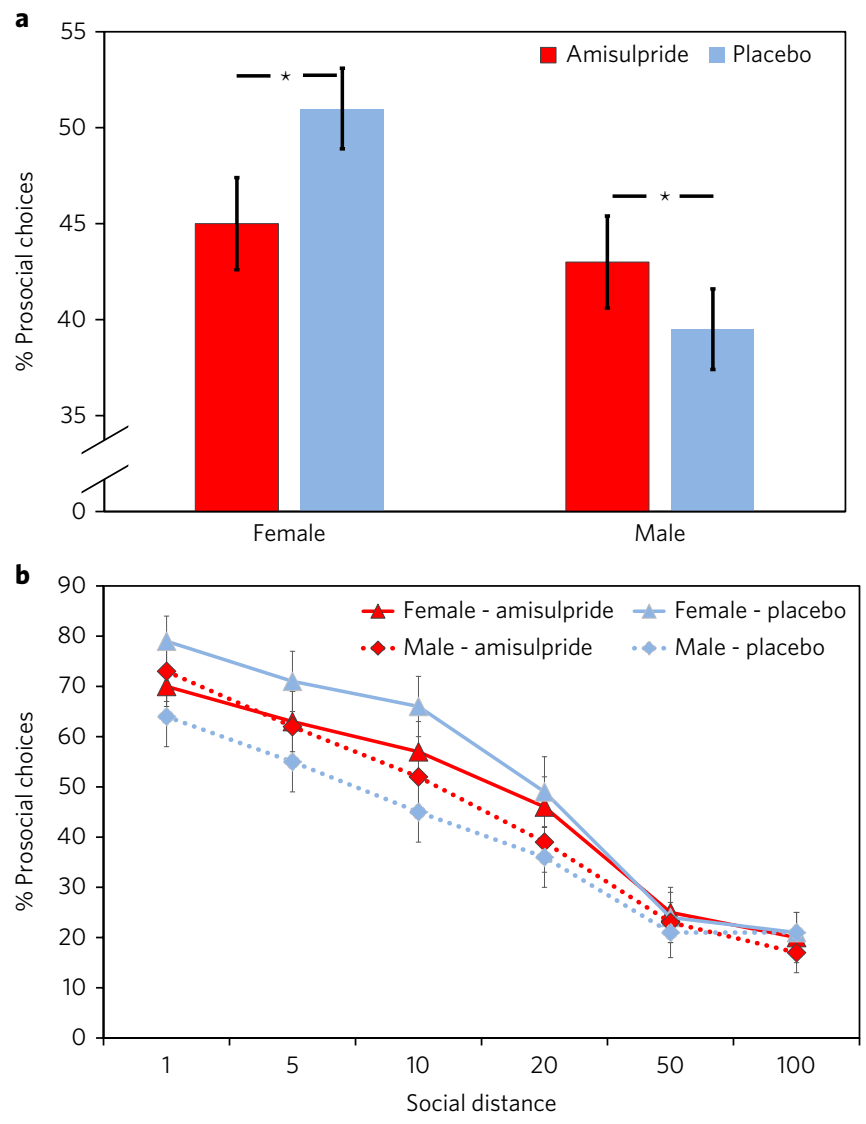

Fig. 2 | Gender-related effects of amisulpride on prosocial choices.

a, Percentage of prosocial choices in the interpersonal decision task $(n=55)$, plotted as a function of gender (female versus male) and substance (amisulpride versus placebo). The asterisk indicates significant differences $(P<0.05$; paired-samples $t$-tests). Error bars indicate within-subject error of the mean difference between amisulpride and placebo, for females and males separately. $\mathbf{b}$, Prosocial choices plotted as a function of gender, substance and social distance. In both females and males, amisulpride affected social preferences predominantly when making decisions about socially close others. Error bars indicate standard error of the mean.

interaction between Substance and Gender also remained significant when controlling for individual differences in BMI as a proxy for the concentration of amisulpride in the blood $(F(1,50)=9.78$, $\left.P=0.004, \eta_{\mathrm{p}}{ }^{2}=0.157\right)$. These findings suggest that the genderrelated drug effects indeed reflect gender-related functioning of the dopaminergic reward systems in social decision-making rather than potentially confounding factors such as baseline prosociality or effective concentration of amisulpride in the blood.

Next, we tested whether dopamine blockade modulates social preferences in general, or whether the effects of amisulpride depend on the social distance of the other person. To assess whether the drug effects on prosociality are dependent on the social distance of the other person, we added the factor Social Distance to our ANOVA model. The significant main effect of Social Distance indicated that participants shared money more often with close rather than distant others $\left(F(5,255)=67.20, P<0.001, \eta_{\mathrm{p}}{ }^{2}=0.569\right)$, consistent with previous findings $s^{18-20}$. Importantly, we again observed a significant Substance $\times$ Gender interaction $(F(1,51)=6.24$, $\left.P=0.016, \eta_{\mathrm{p}}{ }^{2}=0.109\right)$. In addition, this interaction was qualified by a three-way Social Distance $\times$ Substance $\times$ Gender interaction $\left(F(5,255)=2.34, P=0.042, \eta_{\mathrm{p}}{ }^{2}=0.044\right)$ (Fig. 2b). Females decided to share money less often under amisulpride than under placebo with close others at social distances 1, 5 and 10 (all $t>2.09$, all $P<0.047$, all Cohen's $d>0.40$ ), but not with more distant others (all $t<1$, all $P>0.58$, all Cohen's $d<0.11$ ). An analogous pattern was observed in the male sample: relative to placebo, amisulpride rendered males more prosocial towards close others, social distances $1(t(27)=1.92$, $P=0.07$, Cohen's $d=0.36)$ and $5(t(27)=2.04, P=0.05$, Cohen's $d=0.39$ ), but not towards more distant others (all $t<1.30$, all $P>0.20$, all Cohen's $d<0.25$ ). These data suggest that amisulpride modulates social preferences predominantly when confronted with close others. Again, this pattern of findings speaks against the possibility that choices became noisier under amisulpride, because amisulpride differentially affected decisions at low social distances even though both genders more often chose the prosocial than the selfish option under placebo. In both women and men, prosocial choices under placebo were significantly above $50 \%$ at social distance 1 (both $t>2.2$, both $P<0.04$ ), as well as significantly below $50 \%$ at social distances 50 and 100 (all $t>5.5$, all $P<0.001$ ). If choices had become noisier (and thus less determined by an individual's social preferences), both women and men should have shown less prosocial choices at social distance 1 under amisulpride compared with placebo and more prosocial at social distances 50 and 100 (that is, choices should have been closer to the $50 \%$ chance level). However, neither of these predictions was confirmed by the data, because choices at these conditions remained significantly different from chance level under amisulpride (all $t>3.8$, all $P<0.001$ ), and men made even more prosocial choices under amisulpride than under placebo at social distance 1 . Thus, we can rule out the possibility that amisulpride made choices more random instead of modulating social preferences.

As a further test of how the effect of dopamine blockade depends on social distance, we determined the degree of social discounting for each individual participant by fitting a hyperbolic discount function to the individual indifference values in the interpersonal decision task (see Methods). The intercept $V$ measures generosity to socially close others, and the discount factor $s$ measures the steepness of the discounting of shared rewards with increasing social distance. We analysed the parameter estimates (that is, the individually estimated parameters $V$ and $s$ ) as the dependent variable in a mixed-measures ANOVA including the factors Substance (amisulpride versus placebo), Parameter ( $V$ versus $s$ ), Gender (female versus male), and Substance Order (amisulprideplacebo versus placebo-amisulpride). The inclusion of the factor 'Parameter' allowed us to test whether the drug effects were stronger on intercept $V$ than on discount factor $s$. We found significant effects of Parameter $\left(F(1,51)=1,341.33, P<0.001, \eta_{\mathrm{p}}{ }^{2}=0.963\right)$ and Substance $\times$ Gender $\left(F(1,51)=8.92, P=0.001, \eta_{\mathrm{p}}{ }^{2}=0.149\right)$, which were specified by a three-way Parameter $\times$ Substance $\times$ Gender interaction $\left(F(1,51)=8.88, P=0.001, \eta_{\mathrm{p}}{ }^{2}=0.148\right)$ (these effects are robust to mean-centering the parameter estimates for $V$ and $s$ and therefore are not due to scaling differences between $V$ and $s$ ). These results suggest that amisulpride affected parameters $V$ and $s$ differently in the two genders (Fig. 3 and Supplementary Figure 2). More specifically, we found that under amisulpride, relative to placebo, intercept $V$ was significantly reduced in female participants $(t(26)=2.07, P=0.024$, Cohen's $d=0.40)$ and significantly increased in male participants $(t(27)=2.13, P=0.022$, Cohen's $d=0.40)$, while there were no drug effects on discount factor $s$ in either gender (both $t<1$, both $P>0.50$, both Cohen's $d<0.13$ ). These findings specify the above-reported gender-related drug effects on prosocial choices and suggest that, compared to placebo, under amisulpride female and male participants tend to be more selfish and prosocial, respectively, when they make sharing decisions about socially close others.

No evidence for gender-related drug effects on non-social decision-making. To determine whether the observed genderrelated drug effects are specific for social decision-making, we 
a

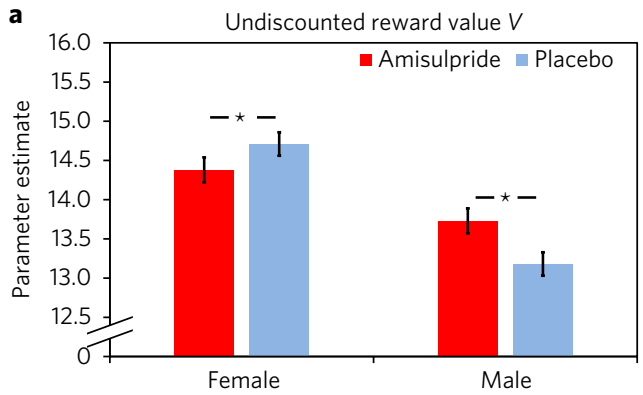

b

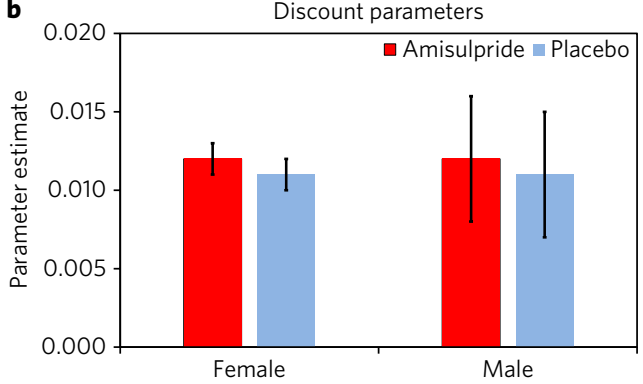

Fig. 3 | Gender-related effects of amisulpride on social discounting parameters. a,b, Parameter estimates for undiscounted reward value $V$ (a) and discount factor $s$ in the interpersonal decision task $(\mathbf{b})(n=55)$, plotted as a function of gender (female versus male) and substance (amisulpride versus placebo). The asterisk indicates significant differences $(P<0.05$; paired-samples $t$-tests). Error bars indicate within-subject error of the mean difference between amisulpride and placebo, for females and males separately.

analysed the impact of amisulpride on choices in the intertemporal decision task. Because previous studies related dopaminergic activity to preference for SS over LL rewards ${ }^{23,24}$, we expected blocking of dopaminergic activity to lead to a higher number of LL choices. More importantly, we expected to find no genderrelated effects of amisulpride on intertemporal choices. In analogy to the interpersonal decision task, we analysed the percentage of LL choices with a mixed-measures ANOVA including the factors Substance (amisulpride versus placebo), Gender (female versus male) and Substance Order (amisulpride-placebo versus placeboamisulpride). Besides an interaction between Gender and Substance Order (which may represent a chance finding; $F(1,51)=6.09$, $P=0.017, \eta_{\mathrm{p}}^{2}=0.107$ ), no effect passed the statistical threshold (all $F<1.20$, all $P>0.28$, all $\eta_{\mathrm{p}}{ }^{2}<0.015$ ), providing no evidence for drug effects on intertemporal choices (Fig. 4a). However, when entering BMI values (as an approximation for the effective dose of amisulpride) as covariate into the analysis, we found a significant main effect of Substance $\left(F(1,50)=4.55, P=0.038, \eta_{\mathrm{p}}{ }^{2}=0.083\right)$, which was modulated by a Substance $\times$ BMI interaction $(F(1,50)=5.05$, $P=0.029, \eta_{\mathrm{p}}{ }^{2}=0.092$ ): the lower the BMI (and thus the higher the concentration of the drug in the body), the stronger the participants' preference for LL rewards under amisulpride than under placebo (Fig. 4b). Importantly, however, the effect of amisulpride did not differ between female and male participants, even when controlling for BMI, because no further comparison was significant (all $F<1.47$, all $P>0.23$, all $\eta_{\mathrm{p}}{ }^{2}<0.028$ ). Thus, as predicted, we found no evidence to suggest gender-related effects of amisulpride on intertemporal decision-making.

To interrogate the hypothesis-conforming non-significant Substance $\times$ Gender interaction in more detail, we compared the likelihoods of the data under the null hypothesis (drug effects are independent of gender) and under the alternative hypothesis
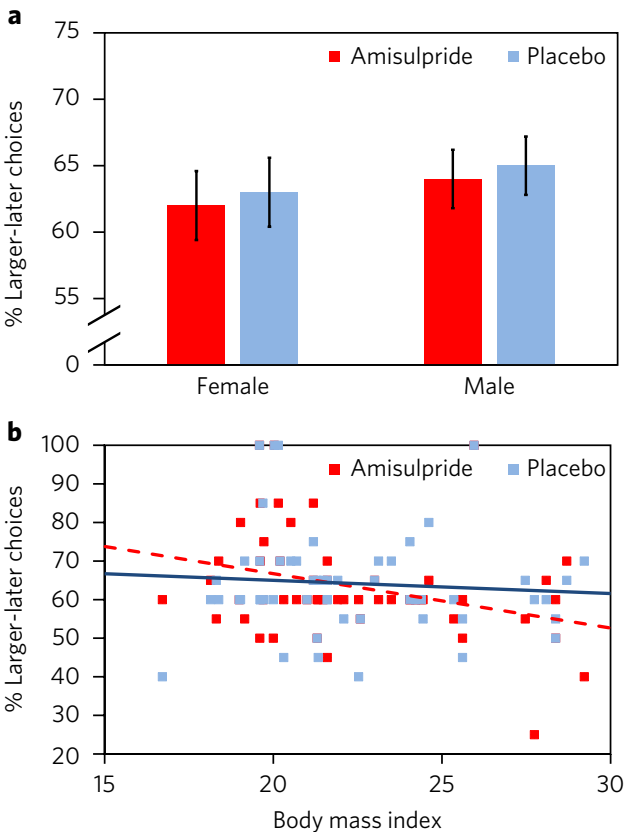

Fig. 4 | Effects of amisulpride on intertemporal choices. a, In the intertemporal decision task $(n=55)$, there were no gender-related drug effects in choices of the larger-later reward. $\mathbf{b}$, Drug effects varied as a function of individual BMI, with low-BMI individuals, who probably had a higher concentration of amisulpride in their blood, being more patient under amisulpride than under placebo (Substance $\times \mathrm{BMI}$ interaction, $F(1,50)=5.05, P=0.029)$. Error bars indicate within-subject error of the mean difference between amisulpride and placebo, for females and males separately.

(gender modulates drug effects) by estimating a Bayes factor using the Bayesian information criterion ${ }^{25,26}$. The estimated Bayes factor suggested that it is 7.40 times more likely to observe the data under the null hypothesis than under the alternative hypothesis, which, according to the guidelines for interpreting Bayes factors ${ }^{27}$, provides substantial support for the null hypothesis of gender-unrelated drug effects on intertemporal choices.

To test the task-specificity of the gender effect more stringently, we directly compared drug effects on intertemporal and interpersonal choices by running a between-task ANOVA including the factors Task (interpersonal versus intertemporal decision task), Substance, Gender, and Substance Order. We also included a between-subject factor for Task Order to test whether drug effects on the decision tasks depended on the order in which participants performed the interpersonal and intertemporal decision tasks. The ANOVA revealed a significant Task $\times$ Substance $\times$ Gender interaction $\left(F(1,47)=8.32, P=0.006, \eta_{\mathrm{p}}{ }^{2}=0.113\right)$, in line with a task-specific gender-related effect of amisulpride. This three-way interaction was independent of potential task order effects $(F<1, P=0.38$, all $\left.\eta_{\mathrm{p}}{ }^{2}=0.016\right)$. These results confirm the findings reported above and suggest that amisulpride showed gender-related effects only in the interpersonal, but not the intertemporal, decision task.

Striatal activation during social decision-making differs between women and men. The results of the pharmacological study suggest dissociable roles of dopaminergic neurotransmission for social decision-making in women and men. Because dopamine plays a central role in value-processing brain regions such as the striatum ${ }^{8,21}$, and dopamine manipulations affect haemodynamic value signals in the striatum ${ }^{28-30}$, our findings predict gender-related striatal activations during social decision-making. If dopaminergic activity is 
more pronounced for prosocial behaviour in women and for selfish behaviour in men, we expect activation in the striatum related to prosocial versus selfish decisions to be stronger in women than men. To test this prediction, we combined data from a previous, hitherto unpublished, functional magnetic resonance imaging (fMRI) experiment ( 8 female and 9 male participants) and from an already published study (11 female and 12 male participants $)^{19}$. In both studies, participants performed the previously described interpersonal decision task in the MRI scanner. Specifically, we examined whether the striatum showed higher activity for prosocial versus selfish choices in women relative to men, using a mask for valueprocessing regions in the striatum based on recent meta-analyses ${ }^{31,32}$ (Fig. 5a). The striatum showed gender-related response differences. In agreement with our hypothesis, striatal activations for prosocial versus selfish decisions were stronger in female participants than in male participants $(t(38)=2.25, P=0.03$, Cohen's $d=0.73$; Fig. $5 b)$. An exploratory whole-brain analysis provided no evidence for gender differences in any further brain region when using familywise error (FWE) correction at the cluster level. At more liberal thresholds $(P<0.001$, number of voxels, $k>5)$, females, relative to males, showed enhanced activation during prosocial versus selfish decisions in the posterior insula (peak coordinates: $x=36, y=-19$, $z=13 ; k=27$ voxels), superior temporal lobe $(x=60, y=-10, z=4$; $k=6$ voxels) and the temporoparietal junction $(x=51, y=-28$, $z=31 ; k=22$ voxels).

In the unpublished (but not in the published) experiment, participants also performed the intertemporal decision task in the scanner. Similar to the pharmacological experiment, this allowed us to test whether the gender differences in the interpersonal decision task are specific for social decision-making. In line with previous reports ${ }^{33}$, the striatum was more active for SS than LL choices across both genders $(t(16)=2.57, P=0.021$, Cohen's $d=0.62$; Fig. 5c). More importantly, striatum activation related to SS versus LL choices did not differ between female and male participants $(t(15)=1.26, P=0.22$, Cohen's $d=0.61$; Fig. $5 c)$. Also, an exploratory whole-brain analysis provided no evidence for neural gender differences during intertemporal choice even at more liberal thresholds $(P<0.001, k>5)$. In contrast, in the interpersonal decision task, the gender difference was significant even when restricting the analysis to the sample of the unpublished experiment $(t(15)=2.24$, $P=0.048$, Cohen's $d=1.16$ ). This gender difference in striatal activation was also independent of different payoff structures of the prosocial reward (see Methods and Supplementary Fig. 3). Thus, despite the small sample size of the unpublished experiment, which should be kept in mind as a caveat, we found significant gender differences that were selective for interpersonal choice. Together, the neuroimaging results mirror the results from the pharmacological experiment and suggest dissociable roles of the neural reward system in women and men for social, but not individual, decision-making.

\section{Discussion}

The current study provides a neurobiological foundation for a welldocumented gender difference in sharing behaviour ${ }^{1,2}$. We find that in females the dopaminergic reward system is more sensitive to shared than to selfish rewards, while the opposite is true for males. This conclusion is evidenced both by the pharmacological and neuroimaging data.

Pharmacologically blocking dopaminergic transmission reduced prosociality in women and selfishness in men. This finding is compatible with the notion that dopamine encodes value and promotes reward seeking ${ }^{7,8,34}$. According to a recent theoretical account on gender differences in social preferences ${ }^{2}$, sharing tends to be more preferable than acting selfishly for women, whereas, for men, maximizing self-reward tends to carry more value. It is important to note that dopaminergic signals may encode the subjective value of both prosocial and selfish reward options in both genders, rather than encoding selectively the value of the prosocial reward option in women and the value of the selfish reward option in men. However, due to the different biases of women and men for prosocial and selfish rewards, respectively, dopaminergic signals may more strongly encode shared (relative to selfish) rewards in women and selfish (relative to shared) rewards in men. Dopamine receptor blockade would then reduce the impact of these aspects on the decision process, in line with our finding of increased selfish choices in women and prosocial choices in men.

The gender-related effects of dopamine receptor blockade are mirrored by the finding that, compared to males, females show enhanced striatal activations during prosocial relative to selfish decisions. Our results are consistent with previous single-gender findings of enhanced striatal activation during costly sharing in women ${ }^{11}$ and of enhanced selfishness after increasing dopaminergic

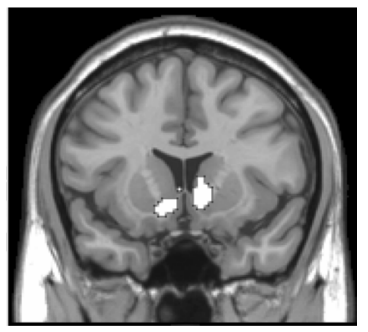

b

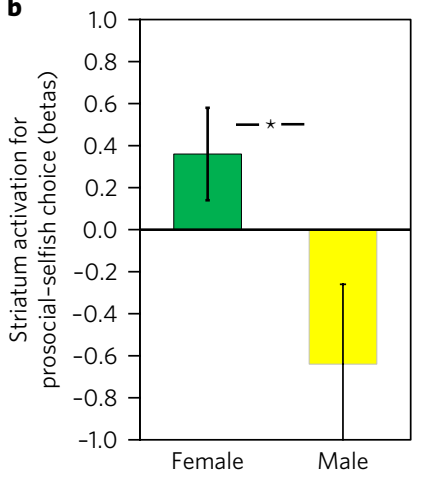

c

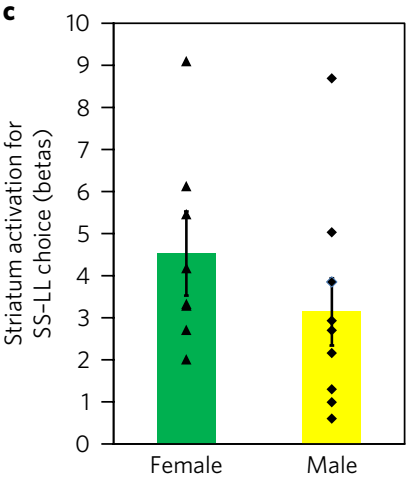

Fig. 5 | Results of the neuroimaging study. a, Pre-defined mask of value-processing regions in the striatum (which served as a region of interest for examining decision-related activity during interpersonal and intertemporal decision tasks. $\mathbf{b}$, In the interpersonal decision task $(n=40)$, female participants showed stronger striatum activation related to choices of prosocial versus selfish rewards than male participants $(t(38)=2.25, P=0.03)$, consistent with the findings of the pharmacological experiment. c, In contrast, in the intertemporal decision task ( $n=17$ ), striatum activation related to choices of SS versus LL rewards did not differ between female and male participants $(t(15)=1.26, P=0.22)$. Because the number of female or male participants was relatively low for this comparison, we display individual data points. The asterisk indicates significant difference $(P<0.05$; independentsamples $t$-tests). Error bars indicate standard error of the mean. 
neurotransmission in $\operatorname{men}^{16}$. The present findings inform and qualify these previous studies by showing the necessity of considering gender differences in the neuroscience of social decision-making and provide a link between dopamine and gender differences in prosocial preferences. Our results are also consistent with the view that gender differences in the brain are functional, rather than morphological, in nature ${ }^{35,36}$.

In contrast to the clear gender-related effects observed in interpersonal decision-making, neither the pharmacological nor the imaging study revealed evidence for gender-differences in the paradigm we used to assess intertemporal decision-making. Although there is some evidence that temporal discounting may differ between women and men $^{37}$, our findings suggest that these potential differences appear to be smaller than in isomorphic social discounting tasks and unrelated to dopaminergic functioning.

It is worth keeping in mind that the currently observed gender differences do not have to be innate or hard-wired. In contrast, because dopaminergic activity plays a role in reward learning and encodes the learned value of actions or stimuli ${ }^{8,29}$, gender-related reward processing may be the result of education and/or cultural expectations ${ }^{4,5}$. Note that we do not assume such cultural expectations or stereotypes to describe gender differences that exist independently of these stereotypes. Instead, these stereotypes might function as self-fulfilling prophecies and produce the gender differences they claim to describe. For example, from an early age, women may receive more positive feedback for prosocial behaviour than men, which may lead to an internalization of cultural norms and make prosocial behaviour more valuable and predictive of rewarding feedback ${ }^{4}$. If true, this notion would suggest that the presently observed effects are not an expression of hard-wired differences between men and women per se, but rather that education and learning history may be the driving factors for differential associations of high reward value to different behaviours by the dopaminergic reward system. Consistent with the assumption of cultural influences on dopaminergic functioning, polymorphisms of dopaminergic receptor genes appear to be associated with more independent social orientation in European Americans and more interdependent social orientation in Asians ${ }^{38}$. Together, experience may shape the sensitivity of the dopamine system to social information.

The observed effects cannot be explained by findings showing that the same dose of amisulpride leads to higher plasma concentrations in the blood of females than of males ${ }^{39}$, or that males have a higher striatal D2 receptor density than females ${ }^{40}$. This is because the gender differences were specific to social decision-making, while non-social tasks showed no gender-related drug effects. It is also worth noting that there were no gender differences in working memory capacity (as a proxy for baseline dopamine levels), so the observed effects cannot be explained by an inverted-U effect of dopamine on cognition (which would, moreover, not have been limited to the domain of social decision-making). Thus, due to the high task-specificity of our findings, a gender-related role of dopamine in prosocial decision making, rather than gender differences in amisulpride absorption per se, offers the most parsimonious explanation for our findings.

Although the actions of amisulpride on D2/D3 receptors are relatively selective, it also blocks serotonergic 5-HT7-receptors ${ }^{41}$. Some evidence in humans suggests that the dopamine and serotonin systems play opposing or at least complementary roles s $^{23,24,42-44}$. In this view, the serotonin system primarily underpins punishment processing, whereas the dopamine system primarily underpins reward processing. However, the specific function of the 5-HT7-receptor in cognitive processes remains largely unknown, except for a role in memory formation, sleep and psychiatric disorders ${ }^{45}$. Activation of 5 -HT7 receptors in rat ventral striatum decreased ambulatory activity but had no effects on reward-related behaviour ${ }^{46}$. On balance, we therefore believe that it is unlikely that the present effects of amisulpride were mediated by 5 -HT7 actions.

To conclude, we have advanced the current understanding of the neurobiological roots of costly giving. By providing evidence for a dissociable role of dopamine in social decision-making for men and women, our data corroborate a recent theoretical account assuming crucial gender differences in social decision-making and offer a biological account of the finding that prosocial behaviour is often more widespread in women than in men.

\section{Methods}

Pharmacological study (Study 1). Participants. A total of 56 participants (27 female, $M_{\text {age }}=23.2$ years, $\mathrm{SD}_{\text {age }}=3.1$ years) were recruited at the University of Zurich to participate in the study. The study protocol was approved by the Research Ethics Committee of the canton of Zurich. All participants gave written informed consent. One male participant was excluded from analysis due to not following task instructions (he did not indicate any persons for the social distance levels 1-20). For their participation, participants were paid CHF30 per hour and a monetary bonus depending on their choices (see 'Experimental design and procedure')

Interpersonal decision task. Participants performed an interpersonal decision task in which they had to choose between a selfish reward option (CHF7.5-15.5) and a prosocial reward option (CHF7.5 for both the participant and a second person $)^{18-20}$. The social distance of the other person ranged from very close others to complete strangers. In more detail, at the start of the experiment (before drug intake), we asked participants to imagine a list of 100 people ranging from 1 (the person socially closest to them) to 100 (a random stranger on the street). A person at rank 50 was described as a person the participants had seen several times without knowing the name of the person. We discouraged participants to think of people they felt negatively towards. In the computer experiment, we used the social distances of $1,5,10,20,50$ and 100 . For each trial of the interpersonal decision task, the amount of the selfish reward option (for example, 'CHF 12.5 self only') and the social distance of the other (for example, 'Person 20') were displayed (Fig. 1b), with one option presented above a central fixation cross and the other below the fixation cross (counterbalanced across participants). Note that the amount of the prosocial reward option was not displayed because it was fixed at CHF7.5 for both. Participants were instructed to press the left or right control key on a QWERTZ keyboard to choose the selfish or prosocial reward option (the assignment of keys to choice options was counterbalanced across participants).

In each trial, the choice options were displayed until participants responded. The next trial started after an inter-trial interval (ITI) of $1 \mathrm{~s}$. Each combination of social distance and selfish reward level was presented once during the experiment, resulting in a total of 54 trials for the interpersonal decision task. Trials were presented in randomized order.

Intertemporal decision task. To test whether gender-related effects of amisulpride on the interpersonal decision task are selective for social decisions, participants also performed an intertemporal decision task as a non-social control task ${ }^{33}$. We decided to use an intertemporal decision task as the control task because, in the literature, the role of dopamine in time preferences has already been welldescribed $^{23,24,42}$. In this task, participants made choices between SS (for example, CHF80 today) and LL reward options (for example, CHF100 in 90 days). We used a dynamic version of this task in which the choice options were individually selected so that the information provided by each decision was optimized (dynamic experiments for estimating preferences, $\mathrm{DEEP}^{47}$ ). Choice options were presented on the left or right screen side, and the screen sides for the SS and LL reward option were counterbalanced across trials. Participants pressed the left or right arrow keys on a QWERTZ keyboard to choose the option presented on the left or right screen side.

On each trial, the reward options were presented until participants made a choice (Fig. 1c). The next choice options were displayed after an ITI, during which a central fixation cross was presented for $1 \mathrm{~s}$. Participants made a total of 20 choices between SS and LL reward options.

Experimental design and procedure. All participants completed two experimental sessions (each taking $4 \mathrm{~h}$ ), which were separated by exactly 14 days. The experiment followed a randomized, double-blind, placebo-controlled crossover design: half of the participants received an oral dose of $400 \mathrm{mg}$ amisulpride (Solian) in the first session and placebo (mannitol) in the second session, and vice versa for the other half of the participants (Fig. 1a). Participants were not aware of whether they received placebo or amisulpride, as assessed by a post-experimental questionnaire $\left(\chi^{2}(1)<1, P=0.76\right)$, in line with previous, independent studies using the same dose of amisulpride $\mathrm{e}^{23,48}$. Before substance intake, participants performed the digit span task forwards and backwards to measure working memory performance as a proxy of baseline dopamine synthesis capacity ${ }^{49,50}$. Moreover, participants performed a mood questionnaire before substance intake and at the end of the experiment to control for potential drug effects on mood. 
The experimental tasks were performed $\sim 1.5 \mathrm{~h}$ after substance intake, that is, $0.5 \mathrm{~h}$ after the first peak in amisulpride plasma level concentration. As well as the interpersonal decision task and the intertemporal decision task, participants performed further experimental tasks measuring risk and effort preferences, a reversal learning task, and a stop-signal task (in counterbalanced order). The results for these tasks will be reported in a separate article. Importantly, none of these tasks showed gender-related drug effects or gender differences under placebo (all $F<2.47$, all $P>0.12$ ).

The task was incentive-compatible. Specifically, participants knew beforehand that, at the end of the experiment, one single trial would be randomly selected and implemented both for the interpersonal decision task and for the intertemporal decision task. If participants had chosen the selfish or SS reward option in the selected trial, they received the corresponding amount of the selfish reward option or SS reward option immediately, and in addition to their basic payment. If participants had chosen the prosocial reward option in the interpersonal decision task, both they and the person at the corresponding social distance received CHF7.5. The contact details of the other person were recorded after the experimental session and the money was sent to the other person by mail. Similarly, if participants had chosen the LL reward in the intertemporal decision task, the corresponding LL amount was sent to the participant after the temporal delay of the chosen option. Participants were explicitly instructed that they should take all decisions seriously, as every decision had equal probability of being chosen at the end and implemented.

Data analysis. Statistical analysis of the behavioural data was performed with Matlab R2014b (MathWorks) and IBM SPSS Statistics 22. For the interpersonal decision task, we computed hyperbolic discount functions reflecting the discounted subjective value of the prosocial reward as a function of social distance, following the procedure taken by previous studies ${ }^{18-20}$. We first calculated individual indifference points using logistic regression predicting choice as a function of selfish reward level. Each indifference point was defined as the value at which a participant would choose the prosocial and the selfish reward option with an equal probability of $50 \%$. These indifference points were computed separately for each social distance. Finally, social discount functions were fitted to the indifference points, separately for each participant. We assumed that social discounting can be described by the following standard hyperbolic function ${ }^{18}$ :

$$
S V=V /(1+s \times D)
$$

where $S V$ is the discounted value of the prosocial reward option, and $V$ is the undiscounted value of the prosocial reward and determines the height of the discount function at social distance 0 (intercept). $D$ indicates the social distance of the other person, and $s$ represents a participant-specific constant measuring the degree of discounting. A larger $s$ corresponds to stronger social discounting, that is, a stronger increase in selfish choices with increasing social distance. The undiscounted reward value $V$ as well as the discount parameter $s$ were free parameters and were determined separately for each participant by fitting equation (1) to their indifference points.

We analysed choices as well as the parameter estimates for $V$ and $s$ in the interpersonal and intertemporal decision tasks with mixed-measures ANOVAs as implemented in SPSS Statistics 22. In addition, we conducted planned comparisons to test the hypothesized gender-related effects of amisulpride on social decisionmaking. The alpha threshold was set to $5 \%$ for all tests.

Neuroimaging study (Study 2). For the neuroimaging study, we collapsed the data from two separate functional imaging experiments in which subjects had performed the interpersonal decision task in the fMRI scanner. The data of the first experiment (neuroimaging experiment 1) were published previously ${ }^{19}$ without having analysed gender differences, whereas the data of the second experiment (neuroimaging experiment 2) have not yet been published. Both studies were conducted before the pharmacological study and did not include any pharmacological manipulation. Combining the data sets from these two studies allowed us to test for gender-related blood-oxygen-level dependent (BOLD) responses in the neural reward system with adequate sample sizes (19 women, 21 men)

Neuroimaging experiment 1. Participants. The sample of the first imaging experiment comprised 11 female and 12 male participants.

Task design and image acquisition. Participants performed the interpersonal decision task in a similar way as in the pharmacological study: participants made choices between selfish reward options (ranging from $€ 75$ to $€ 165$ ) and sharing money with other persons at varying social distances. Similar to the pharmacological study, the amounts of the prosocial reward option were fixed at $€ 75$ for the subject and the other (for details and a description of image acquisition, see ref. ${ }^{19}$ ).

Functional imaging analysis. All imaging analyses were performed with SPM 12 (www.fil.ion.ucl.ac.uk/spm). We tested whether the striatum shows gender-related effects in the general linear model (GLM), which included two separate onset regressors of main interest, one for prosocial decisions and one for selfish decisions in the interpersonal decision task, modelled at the corresponding decision times. The GLM also included onset regressors for the presentation of the social distance, the prosocial reward option and the selfish reward option, as well as six movement parameters as covariates of no interest. All regressors were convolved with the canonical haemodynamic response function. We then extracted regression parameters comparing activity related to prosocial versus selfish choices in every voxel within a predefined region of interest (ROI; see next section) for each participant. Gender differences in the extracted beta values were tested with independent-samples $t$-tests. In addition to the ROI analysis, we performed an exploratory whole-brain analysis by estimating regression parameters in every voxel for each participant. We then entered the parameter estimates in a betweenparticipant, random-effects analysis to obtain statistical parametric maps for independent-sample $t$-tests comparing activity related to prosocial versus selfish choices. We used family-wise error correction at the cluster level to correct for multiple comparisons $(P<0.05$, with a cluster-inducing threshold of $P<0.001)$.

ROI definition. We focused on the striatal regions where activity correlated with subjective value, as recently identified in independent meta-analyses ${ }^{31,32}$. This mask is based on the conjunction of all voxels in the striatum showing a significant positive correlation with the value of rewards during decision-making in these meta-analyses, and can be downloaded from http://www.rnl.caltech.edu/resources/ index.html.

Neuroimaging experiment 2. Participants. Seventeen volunteers ( 8 female and 9 male; $\left.M_{\text {age }}=23.7 ; \mathrm{SD}_{\text {age }}=5.6\right)$ participated in the study after giving written informed consent. The study was approved by the Research Ethics Committee of Zurich. Participants received CHF50 and a monetary bonus depending on their choices (see next section).

Task design and procedure. Participants performed the interpersonal and intertemporal decision tasks in a similar way as in study 1 . In the interpersonal decision task, the height of the selfish reward option varied from CHF65 to CHF155 in steps of 10 . For the prosocial reward option, three generosity levels were used: CHF75 for self and CHF75 for the other, CHF75 for self and CHF25 for the other, CHF100 for self and CHF50 for the other. As the current study focused on a comparison between choices of the prosocial and the selfish reward option, we collapsed data across different generosity levels for the following analyses.

Each trial of the interpersonal decision task started with a presentation of the social distance information. Then, either the selfish or prosocial reward option was presented, followed by the second choice option. The social distance information was presented first (2-3s), followed by the two choice options (also 2-3s). Next, participants had $3 \mathrm{~s}$ to indicate their response by pressing a left or right response key for the option presented on the left or right screen side, respectively. At the end of each trial, a feedback indicating the choice was shown for $0.5 \mathrm{~s}$. The minimum ITI was $2 \mathrm{~s}$ and was jittered with a Poisson distribution (maximum ITI of $13 \mathrm{~s}$ ). Consequently, the total length of a trial varied from 11.5 to $25.5 \mathrm{~s}$.

In the intertemporal decision task, participants chose between SS and LL rewards. The SS reward ranged from CHF30 to CHF150 in steps of 30, which resulted in five SS reward levels. The LL reward was held constant at CHF160, with the temporal delay varying from 3 to 15 months in steps of 3 , which resulted in five delays.

For each trial, we first presented either the SS reward option or the LL reward option, followed by the corresponding alternative option. As in the interpersonal decision task, the choice options were presented for 2-3 s. After the presentation of the second option, the decision period started $(3 \mathrm{~s})$, in which participants had to indicate their response by pressing a left or right response key for the option presented on the left or right screen side, respectively. Feedback was presented for $0.5 \mathrm{~s}$. The ITI ranged from $2 \mathrm{~s}$ to $13 \mathrm{~s}$, resulting in a total trial length from 9.5 to $22.5 \mathrm{~s}$.

Participants performed five runs, each including 42 interpersonal decisions and 20 intertemporal decisions. The task order within each run was randomized across participants. As in the pharmacological experiment, one trial in each task was randomly selected at the end of the experiment and implemented.

Image acquisition. The fMRI sessions were performed at the Laboratory for Social and Neural Systems Research (SNS lab) at the University Hospital of Zurich. The MRI system used was a $3 \mathrm{~T}$ Philips Achieva whole-body scanner equipped with an eight-channel SENSE head coil (Philips Medical Systems). The functional images were acquired using a BOLD-T2*-weighted single-shot echo-planar imaging (EPI) pulse sequence. The acquisition parameters were determined as follows: $T_{\mathrm{E}}=30 \mathrm{~ms}$, $T_{R}=1,565 \mathrm{~ms}$, with a flip angle of $75^{\circ}$, field of view $(F O V)=240 \times 216 \times 119 \mathrm{~mm}^{2}$, acquisition matrix $=80 \times 72$, voxel size $=3 \times 3 \times 3 \mathrm{~mm}^{3}$, with a slice gap of $1 \mathrm{~mm}$. The anatomical $T_{1}$-weighted images were obtained after the decision tasks were completed using a turbo field echo (TFE) pulse sequence with a flip angle of $8^{\circ}$. The acquisition matrix ranged over $228 \times 227$ with an FOV of $250 \times 250 \mathrm{~mm}^{2}$ and a voxel size of $1.1 \times 1.1 \times 0.6 \mathrm{~mm}^{3}$. 
Functional imaging analysis. The functional data of each participant were slice-timing-corrected, motion-corrected, unwarped, and co-registered to the anatomical image. Following segmentation, we spatially normalized the data into standard Montreal Neurological Institute (MNI) space. Finally, data were smoothed with an $8 \mathrm{~mm}$ full-width at half-maximum (FWHM) Gaussian kernel and high-pass-filtered during statistical analysis.

We analysed the data for the interpersonal decision task with a GLM identical to that used for the first neuroimaging experiment. This allowed us to collapse the data from the current sample with the data from the previous study ${ }^{19}$. A similar GLM was set up to analyse data in the intertemporal decision task. This GLM included two onset regressors of main interest, one for choices of the SS reward option and one for choices of the LL reward option at decision time. In addition, the model included onset regressors for presentation of the SS and LL reward options, as well as movement parameters as regressors of no interest. Again, we used the predefined mask for the striatum to extract the beta values contrasting prosocial versus selfish decisions in the interpersonal decision task and SS versus LL reward choices in the intertemporal decision task. Finally, we collapsed the data for the interpersonal decision task from imaging experiment 1 and imaging experiment 2 and tested for gender differences in the functional striatum mask with independent-samples $t$-tests. Because we combined data from two different scanners, we added a dummy-coded covariate (' 0 ' for neuroimaging experiment 1 and ' 1 ' for neuroimaging experiment 2) to the GLM for the second-level analysis of the interpersonal decision task in order to control for potential effects of the different scanners.

Data availability. The data that support the findings of this study are available from the corresponding author upon request.

Received: 8 March 2017; Accepted: 12 September 2017; Published online: 9 October 2017

\section{References}

1. Croson, R. \& Gneezy, U. Gender differences in preferences. J. Econ. Lit. 47, 448-474 (2009).

2. Rand, D. G., Brescoll, V. L., Everett, J. A., Capraro, V. \& Barcelo, H. Social heuristics and social roles: intuition favors altruism for women but not for men. J. Exp. Psychol. Gen. 145, 389-396 (2016).

3. Rand, D. G. Social dilemma cooperation (unlike dictator game giving) is intuitive for men as well as women. J. Exp. Soc. Psychol. 73, 164-168 (2017).

4. Heilman, M. E. \& Chen, J. J. Same behavior, different consequences: reactions to men's and women's altruistic citizenship behavior. J. Appl. Psychol. 90, 431-441 (2005).

5. Eagly, A. H. Sex Differences in Social Behavior: A Social-role Interpretation (L. Erlbaum Associates, Hillsdale, NJ, 1987).

6. Eagly, A. H. \& Crowley, M. Gender and helping behavior: a meta-analytic review of the social psychological literature. Psychol. Bull. 100, 283-308 (1986)

7. Schultz, W. Multiple dopamine functions at different time courses. Annu. Rev. Neurosci. 30, 259-288 (2007).

8. Schultz, W. Neuronal reward and decision signals: from theories to data. Physiol. Rev. 95, 853-951 (2015).

9. Saez, I., Zhu, L., Set, E., Kayser, A. \& Hsu, M. Dopamine modulates egalitarian behavior in humans. Curr. Biol. 25, 912-919 (2015).

10. Tricomi, E., Rangel, A., Camerer, C. F. \& O’Doherty, J. P. Neural evidence for inequality-averse social preferences. Nature 463, 1089-1091 (2010).

11. Harbaugh, W. T., Mayr, U. \& Burghart, D. R. Neural responses to taxation and voluntary giving reveal motives for charitable donations. Science 316, 1622-1625 (2007).

12. Mobbs, D. et al. A key role for similarity in vicarious reward. Science 324, 900 (2009)

13. Fareri, D. S., Niznikiewicz, M. A., Lee, V. K. \& Delgado, M. R. Social network modulation of reward-related signals. J. Neurosci. 32, 9045-9052 (2012).

14. Hsu, M., Anen, C. \& Quartz, S. R. The right and the good: distributive justice and neural encoding of equity and efficiency. Science 320, 1092-1095 (2008).

15. Morelli, S. A., Sacchet, M. D. \& Zaki, J. Common and distinct neural correlates of personal and vicarious reward: a quantitative meta-analysis. NeuroImage 112, 244-253 (2015)

16. Pedroni, A., Eisenegger, C., Hartmann, M. N., Fischbacher, U. \& Knoch, D. Dopaminergic stimulation increases selfish behavior in the absence of punishment threat. Psychopharmacology 231, 135-141 (2014).

17. Rosenzweig, P. et al. A review of the pharmacokinetics, tolerability and pharmacodynamics of amisulpride in healthy volunteers. Hum. Psychopharmacol. 17, 1-13 (2002).

18. Jones, B. \& Rachlin, H. Social discounting. Psychol. Sci. 17, 283-286 (2006).

19. Strombach, T. et al. Social discounting involves modulation of neural value signals by temporoparietal junction. Proc. Natl Acad. Sci. USA 112, 1619-1624 (2015)

20. Soutschek, A., Ruff, C. C., Strombach, T., Kalenscher, T. \& Tobler, P. N. Brain stimulation reveals crucial role of overcoming self-centeredness in self-control. Sci. Adv. 2, e1600992 (2016).
21. Wise, R. A. Dopamine, learning and motivation. Nat. Rev. Neurosci. 5 , 483-494 (2004).

22. Strombach, T., Margittai, Z., Gorczyca, B. \& Kalenscher, T. Gender-specific effects of cognitive load on social discounting. PloS ONE 11, e0165289 (2016).

23. Weber, S. C. et al. Dopamine D2/3- and $\mu$-opioid receptor antagonists reduce cue-induced responding and reward impulsivity in humans. Transl. Psychiatr. 6, e850 (2016).

24. Kayser, A. S., Allen, D. C., Navarro-Cebrian, A., Mitchell, J. M. \& Fields, H. L. Dopamine, corticostriatal connectivity, and intertemporal choice. J. Neurosci. 32, 9402-9409 (2012).

25. Wagenmakers, E. J. A practical solution to the pervasive problems of $p$ values. Psychonom. Bull. Rev. 14, 779-804 (2007).

26. Jarosz, A. \& Wiley, J. What are the odds? A practical guide to computing and reporting Bayes factors. J. Problem Solving 7, 2 (2014).

27. Jeffreys, H. Theory of Probability 3rd edn (Oxford Univ. Press, New York, NY, 1961).

28. Pine, A., Shiner, T., Seymour, B. \& Dolan, R. J. Dopamine, time, and impulsivity in humans. J. Neurosci. 30, 8888-8896 (2010).

29. Pessiglione, M., Seymour, B., Flandin, G., Dolan, R. J. \& Frith, C. D. Dopamine-dependent prediction errors underpin reward-seeking behaviour in humans. Nature 442, 1042-1045 (2006).

30. Ferenczi, E. A. et al. Prefrontal cortical regulation of brainwide circuit dynamics and reward-related behavior. Science 351, aac9698 (2016)

31. Bartra, O., McGuire, J. T. \& Kable, J. W. The valuation system: a coordinatebased meta-analysis of BOLD fMRI experiments examining neural correlates of subjective value. NeuroImage 76, 412-427 (2013).

32. Clithero, J. A. \& Rangel, A. Informatic parcellation of the network involved in the computation of subjective value. Soc. Cogn. Affect. Neurosci. 9 , 1289-1302 (2014).

33. McClure, S. M., Laibson, D. I., Loewenstein, G. \& Cohen, J. D. Separate neural systems value immediate and delayed monetary rewards. Science 306, 503-507 (2004)

34. Bodi, N. et al. Reward-learning and the novelty-seeking personality: a between- and within-subjects study of the effects of dopamine agonists on young Parkinson's patients. Brain 132, 2385-2395 (2009).

35. Joel, D. et al. Sex beyond the genitalia: the human brain mosaic. Proc. Natl Acad. Sci. USA 112, 15468-15473 (2015).

36. Glezerman, M. Yes, there is a female and a male brain: morphology versus functionality. Proc. Natl Acad. Sci. USA 113, E1971 (2016).

37. Dittrich, M. \& Leipold, K. Gender differences in time preferences. Econ. Lett. 122, 413-415 (2014)

38. Kitayama, S. et al. The dopamine D4 receptor gene (DRD4) moderates cultural difference in independent versus interdependent social orientation. Psychol. Sci. 25, 1169-1177 (2014).

39. Bergemann, N., Kopitz, J., Kress, K. R. \& Frick, A. Plasma amisulpride levels in schizophrenia or schizoaffective disorder. Eur. Neuropsychopharmacol. 14, 245-250 (2004)

40. Andersen, S. L. \& Teicher, M. H. Sex differences in dopamine receptors and their relevance to ADHD. Neurosci. Biobehav. Rev. 24, 137-141 (2000).

41. Abbas, A. I. et al. Amisulpride is a potent 5-HT7 antagonist: relevance for antidepressant actions in vivo. Psychopharmacology 205, 119-128 (2009).

42. Joutsa, J. et al. Dopaminergic function and intertemporal choice. Transl. Psychiatr. 5, e520 (2015).

43. Geurts, D. E., Huys, Q. J., den Ouden, H. E. \& Cools, R. Serotonin and aversive Pavlovian control of instrumental behavior in humans. J. Neurosci. 33, 18932-18939 (2013).

44. Hebart, M. N. \& Glascher, J. Serotonin and dopamine differentially affect appetitive and aversive general Pavlovian-to-instrumental transfer. Psychopharmacology 232, 437-451 (2015).

45. Gasbarri, A. \& Pompili, A. Serotonergic 5-HT7 receptors and cognition. Rev. Neurosci. 25, 311-323 (2014)

46. Clissold, K. A., Choi, E. \& Pratt, W. E. Serotonin 1A, 1B, and 7 receptors of the rat medial nucleus accumbens differentially regulate feeding, water intake, and locomotor activity. Pharmacol. Biochem. Behav. 112, 96-103 (2013).

47. Toubia, O., Johnson, E., Evgeniou, T. \& Delquie, P. Dynamic experiments for estimating preferences: an adaptive method of eliciting time and risk parameters. Manage. Sci. 59, 613-640 (2013).

48. Kahnt, T. \& Tobler, P. N. Dopamine regulates stimulus generalization in the human hippocampus. eLife 5, e12678 (2016).

49. Cools, R., Gibbs, S. E., Miyakawa, A., Jagust, W. \& D’Esposito, M. Working memory capacity predicts dopamine synthesis capacity in the human striatum. J. Neurosci. 28, 1208-1212 (2008).

50. Landau, S. M., Lal, R., O’Neil, J. P., Baker, S. \& Jagust, W. J. Striatal dopamine and working memory. Cereb. Cortex 19, 445-454 (2009).

\section{Acknowledgements}

The authors thank L. Horvath and K. Treiber for help with data collection. This work was supported by grants PP00P1_128574, PP00P1_150739, 00014_165884, CRSII3_141965 
(PNT) and 320030143443 (ARB; PIs: C. Ruff and T. Hare) from the Swiss National Science Foundation, a research credit of the University of Zurich to C.J.B. (FK-16-016) and a Marie Curie International Incoming Fellowship PIIF-GA-2012-327196 to A.R.B. All funders had no role in study design, data collection and analysis, decision to publish or preparation of the manuscript.

\section{Author contributions}

A.S., C.J.B., A.R.B., S.C.W., B.W., T.K. and P.N.T. designed the study. A.S., C.J.B., A.R.B. R.S., J.t.V. and H.H. performed the research. A.S., C.J.B. and I.I.K. analysed the data. A.S. and P.N.T. wrote the manuscript. C.J.B., A.R.B., R.S., S.C.W., I.I.K., J.t.V., H.H., B.W. and T.K. edited and approved the final version of the manuscript.

\section{Competing interests}

The authors declare no competing interests.

\section{Additional information}

Supplementary information is available for this paper at https://doi.org/10.1038/ s41562-017-0226-y.

Reprints and permissions information is available at www.nature.com/reprints. Correspondence and requests for materials should be addressed to A.S.

Publisher's note: Springer Nature remains neutral with regard to jurisdictional claims in published maps and institutional affiliations. 


\section{natureresearch}

Corresponding author(s): Alexander Soutschek

\section{Life Sciences Reporting Summary}

Initial submission $\square$ Revised version $\bigotimes$ Final submission

Nature Research wishes to improve the reproducibility of the work that we publish. This form is intended for publication with all accepted life science papers and provides structure for consistency and transparency in reporting. Every life science submission will use this form; some list items might not apply to an individual manuscript, but all fields must be completed for clarity.

For further information on the points included in this form, see Reporting Life Sciences Research. For further information on Nature Research policies, including our data availability policy, see Authors \& Referees and the Editorial Policy Checklist.

\section{- Experimental design}

\section{Sample size}

Describe how sample size was determined.

2. Data exclusions

Describe any data exclusions.

\section{Replication}

Describe whether the experimental findings were reliably reproduced.

\section{Randomization}

Describe how samples/organisms/participants were allocated into experimental groups.

\section{Blinding}

Describe whether the investigators were blinded to group allocation during data collection and/or analysis.
We planned to test approx. 30 participants per group (females vs. males). Similar samples sizes per experimental group have been used by previous studies that investigated either amisulpride effects on decision-making (Kahnt, Weber, Haker, Robbins, Tobler, 2015, Journal of Neuroscience) or gender differences in social behaviour (Strombach, Margittai, Gorczyca, \& Kalenscher, 2016, PLOS One).

As described in the manuscript, one participants was excluded due to not following task instructions.

No replication experiments were conducted for the experiments described in the manuscript. However, the manuscript contains all information necessary to conduct such replication experiments.

The study followed a double-blind design, participants randomly received either the active substance or placebo in the first session.

The study was double-blind, so neither the experimenters nor the participants knew which substance they received.

Note: all studies involving animals and/or human research participants must disclose whether blinding and randomization were used.

\section{Statistical parameters}

For all figures and tables that use statistical methods, confirm that the following items are present in relevant figure legends (or in the Methods section if additional space is needed).

n/a Confirmed

\The exact sample size $(n)$ for each experimental group/condition, given as a discrete number and unit of measurement (animals, litters, cultures, etc.)

A description of how samples were collected, noting whether measurements were taken from distinct samples or whether the same

sample was measured repeatedly

$\bigotimes$ A statement indicating how many times each experiment was replicated

The statistical test(s) used and whether they are one- or two-sided (note: only common tests should be described solely by name; more complex techniques should be described in the Methods section)

$\square$ \ A description of any assumptions or corrections, such as an adjustment for multiple comparisons

$\square$ \.he test results (e.g. $P$ values) given as exact values whenever possible and with confidence intervals noted

$\square \bigotimes$ A clear description of statistics including central tendency (e.g. median, mean) and variation (e.g. standard deviation, interquartile range)

$\square$ \Clearly defined error bars 
Policy information about availability of computer code

\section{Software}

Describe the software used to analyze the data in this study.

\section{Matlab and SPSS}

For manuscripts utilizing custom algorithms or software that are central to the paper but not yet described in the published literature, software must be made available to editors and reviewers upon request. We strongly encourage code deposition in a community repository (e.g. GitHub). Nature Methods guidance for providing algorithms and software for publication provides further information on this topic.

\section{- Materials and reagents}

Policy information about availability of materials

\section{Materials availability}

Indicate whether there are restrictions on availability of unique materials or if these materials are only available for distribution by a for-profit company.

\section{Antibodies}

Describe the antibodies used and how they were validated for use in the system under study (i.e. assay and species).

10. Eukaryotic cell lines

a. State the source of each eukaryotic cell line used.

b. Describe the method of cell line authentication used.

c. Report whether the cell lines were tested for mycoplasma contamination.

d. If any of the cell lines used are listed in the database of commonly misidentified cell lines maintained by ICLAC, provide a scientific rationale for their use.

There are no restrictions on availability.

No antibodies were used.

Does not apply.

Does not apply.

Does not apply.

Does not apply.

\section{- Animals and human research participants}

Policy information about studies involving animals; when reporting animal research, follow the ARRIVE guidelines

\section{Description of research animals}

Provide details on animals and/or animal-derived materials used in the study.
Does not apply.

Policy information about studies involving human research participants

\section{Description of human research participants}

Describe the covariate-relevant population characteristics of the human research participants.
The samples of female and male participants were matched with regard to age ( mean $=23.2$ years), body mass index, and working memory capacity. 


\section{natureresearch}

Corresponding author(s): Alexander Soutschek

\section{MRI Studies Reporting Summary}

Form fields will expand as needed. Please do not leave fields blank.

\section{- Experimental design}

1. Describe the experimental design.

2. Specify the number of blocks, trials or experimental units per session and/or subject, and specify the length of each trial or block (if trials are blocked) and interval between trials.

3. Describe how behavioral performance was measured.

- Acquisition

4. Imaging

a. Specify the type(s) of imaging.

fMRI

b. Specify the field strength (in Tesla).

c. Provide the essential sequence imaging parameters.

d. For diffusion MRI, provide full details of imaging parameters.

5. State area of acquisition.

whole brain
Exeriment 1: event-related design

Experiment 2: mixed event-related and blocked design

Experiment 1: 160 trials, mean $\mathrm{ITI}=6 \mathrm{~s}$

Experiment 2: participants performed 5 runs, each with 42 trials for interpersonal decision task and 20 trials for intertemporal decision task trial length 9.5-22.5 s (mean 11.5s)

We assessed choices (prosocial vs. selfish or larger-later vs. smallersooner) in the decision tasks.

\section{- Preprocessing}

6. Describe the software used for preprocessing.

7. Normalization

a. If data were normalized/standardized, describe the approach(es).

b. Describe the template used for normalization/ transformation.

8. Describe your procedure for artifact and structured noise removal.

9. Define your software and/or method and criteria for volume censoring, and state the extent of such censoring.
SPM

spatial normalization of functional to individual structural data, then transformation into $\mathrm{MNI}$ space

MNI space

in Experiment 1, three participants were removed due to excessive head movement ( $>4 \mathrm{~mm}$ ). In Experiment 2, no data were removed.

does not apply. 
10. Define your model type and settings.

11. Specify the precise effect tested.

\section{Analysis}

a. Specify whether analysis is whole brain or ROI-based.

b. If ROI-based, describe how anatomical locations were determined.

13. State the statistic type for inference. (See Eklund et al. 2016.)

14. Describe the type of correction and how it is obtained for multiple comparisons.

\section{Connectivity}

a. For functional and/or effective connectivity, report the measures of dependence used and the model details.

b. For graph analysis, report the dependent variable and functional connectivity measure.

16. For multivariate modeling and predictive analysis, specify independent variables, features extraction and dimension reduction, model, training and evaluation metrics.
General linear model (GLM) with onset regressors for prosocial and selfish decisions as regressors of main interest (in Experiment 2, also regressors for smaller-sooner vs. larger-later reward choices). The GLM included further onset regressors modelling the presentation of the different choice options, as well as movement regressors.

Gender-difference in striatum activity related to prosocial vs. selfish decisions.

Both whole brain and ROI (striatum).

ROI for striatum was based on previous meta-analyses.

For the exploratory whole brain analysis, we used a cluster-inducing threshold of $p<0.001, k>5$.

For the exploratory whole brain analysis, no correction for multiple comparisons was used.

Does not apply

Does not apply.

Does not apply. 